\title{
Urgent excision of primary intracardiac Burkitt Lymphoma in a child
}

\author{
Amr Ashry ${ }^{1}$, Abdulla Tarmahomed ${ }^{1}$, Avishek Samaddar ${ }^{1}$, Ramesh Kutty $^{1}$, Ramana \\ Dhannapuneni $^{1}$, Attilio Lotto ${ }^{1}$, and rafael Guerrero ${ }^{1}$ \\ ${ }^{1}$ Alder Hey Children's Hospital
}

October 4, 2021

\begin{abstract}
We present the case of a 9-year-old girl who was referred to our service with a recent history of shortness of breath on exertion and fatigue in addition to visual disturbances and gastrointestinal symptoms. A Transthoracic Echocardiography demonstrated the presence of 3 large intracardiac masses in both the right and left atrium protruding into the mitral and tricuspid valve orifices causing bilateral inflow obstruction. The patient underwent urgent surgical excision of the masses which was uncomplicated. Histological testing was consistent with a rare intracardiac location of Burkitt's Lymphoma.
\end{abstract}

\section{Case Report}

Urgent excision ofprimary intracardiac Burkitt Lymphoma in a child

Amr Ashry* ${ }^{1,2}$, Abdulla Tarmahomed ${ }^{* 3}$, Avishek Samaddar ${ }^{1}$, Ramesh Kutty ${ }^{1}$, Ramana Dhannapuneni ${ }^{1}$, Attilio Lotto ${ }^{1,4}$, Rafael Guerrero ${ }^{1,5}$

1. Department of Paediatric Cardiac Surgery, Alder Hey Children Hospital, Liverpool, United Kingdom.

2. Department of Cardiothoracic Surgery, Assiut University Hospital, Faculty of Medicine, Assiut University, Assiut, Egypt.

3. Department of paediatric cardiology, Alder Hey Children Hospital, Liverpool, United Kingdom.

4. Faculty of health, Liverpool John Moores University, Liverpool, UK.

5. Faculty of Health and Life Science, University of Liverpool, Liverpool UK

\section{Corresponding Author:}

Amr Ashry (MD)

Senior Clinical Fellow at Alder Hey Children Hospital, Liverpool, United Kingdom.

Lecturer of Cardiothoracic Surgery, Faculty of Medicine, Assiut University, Assiut, Egypt.

Address: E Prescot Road, Liverpool, L14 5AB, United Kingdom.

Phone number: +447512622025

E-mail: amr.ashry@aun.edu.eg

Key words: Cardiac tumor, Burkitt Lymphoma, primary cardiac lymphoma, chemotherapy.

Word count: 
Total number of words: 1271

\begin{abstract}
:
We present the case of a 9-year-old girl who was referred to our service with a recent history of shortness of breath on exertion and fatigue in addition to visual disturbances and gastrointestinal symptoms. A Transthoracic Echocardiography demonstrated the presence of 3 large intracardiac masses in both the right and left atrium protruding into the mitral and tricuspid valve orifices causing bilateral inflow obstruction. The patient underwent urgent surgical excision of the masses which was uncomplicated. Histological testing was consistent with a rare intracardiac location of Burkitt's Lymphoma.
\end{abstract}

\title{
Background:
}

Primary intracardiac tumours are quite uncommon. The majority of them are benign, with left atrial myxoma being the most common histological type (1-2). Sarcoma is the most common primary malignant heart tumor (3). Primary cardiac lymphoma (PCL) is even rarer, accounting for only $0.05 \%$ of all primary cardiac tumours (4). We present a rare case of primary intracardiac Burkitt's Lymphoma in a 9 years old child.

\section{Case report:}

A 9-year-old girl with history of asthma and migraine presented with a short history of unspecified gastrointestinal symptoms and diarrhoea. She had increasing severity and frequency of migraine for the last 2 months with a normal MRI of the head in 2019. She also complained of reduced exercise tolerance including fatigue with shortness of breath on exertion. After an Echocardiography performed at the referring hospital showing the presence of intracardiac masses, she was referred to our Cardiac Services at Alder Hey Children Hospital for further assessment and treatment.

On presentation, examination revealed reduced air entry over both lung bases and a loud apical/left sternal ejection systolic murmur and good volume peripheral pulses. Her abdomen was not tender but distended and dull to percussion (ascites) with a $3 \mathrm{~cm}$ hepatomegaly.

Detailed transthoracic echocardiography revealed mild pericardial effusion and 3 heterogenous mobile masses in the both atriums. The right atrial mass measured about $5.6 \times 2.9 \mathrm{~cm}$ in diameter appeared to be attached to atrial septum and protruding through the tricuspid valve in diastole causing severe inflow obstruction. The left atrium had two masses, one of about $2.9 \times 2.6 \mathrm{~cm}$ attached to atrial septum near the mitral annulus occluding the mitral valve in diastole causing severe inflow obstruction. Finally, A second mass, of around $1.8 \times 0.9 \mathrm{~cm}$, was attached to the posterior wall near right lower pulmonary vein origin. Figures 1 and 2 of the TTE show the 3 masses in the 4 chambers view.

Abdominal ultrasound revealed moderate ascites. Chest X-Ray showed bilateral mild pleural effusion, prominence of the central pulmonary vascular markings and the cardiothoracic ratio was at the upper limit of normal. An urgent multidisciplinary team (MDT) meeting was established and, due to the masses causing bilateral severe inflow obstruction, an indication for urgent surgical resection of the intracardiac masses was given.

\section{Intraoperative findings:}

At operation, the patient become haemodynamically unstable during the anaesthetic induction. Following a median sternotomy, upon opening the pericardium a significant pericardial effusion and bilateral pleural effusion were found and drained. The heart presented with significant biatrial dilation. After systemic heparinisation, cardiopulmonary bypass $(\mathrm{CPB})$ was achieved with bicaval and aortic cannulation with a no touch technique to avoid intracardiac embolization. CPB was run at normothermia and after aortic cross clamping, cold blood cardioplegia was infused to achieve cardiac arrest. A right atriotomy was performed and internal inspection of the heart revealed a giant intraatrial tumour, that appeared gelatinous and friable. The mass had a stalk attached to the interatrial septum in correspondence to the coronary sinus blocking the inflow to the tricuspid valve. There was a further attachment to the septal leaflet of the tricuspid valve. 
With careful dissection, the right intra-atrial mass was carefully removed and detached from its adherence to the lower part of the septum, tricuspid valve septal leaflet and coronary sinus. It appeared during dissection that the mass was invading the interatrial septum.

Following this, the interatrial septum was opened within the oval fossa with a longitudinal incision extending it till the left atrial mass was identified which was attached to the opposite side of the lower part of the interatrial septum, in correspondence to the coronary sinus and partially attached to the posterior leaflet of the mitral valve.

The mass was detached from the lower part of the septum and from the mitral valve apparatus. A third firm left atrial mass was removed from the area between the right lower and right upper pulmonary veins. Following this, wash-out of the left and right cavities was performed with normal saline. The interatrial septum was closed with a bovine pericardial patch using continuous 5-0 polypropylene suture. After the right atriotomy was closed with continuous suture, the heart was deaired, ventilation restarted, and cross clamp removed with the heart regaining spontaneously normal sinus rhythm. Figures 3-6 show the intracardiac masses.

A transoesophageal echocardiogram was performed which demonstrated good biventricular function, absence of tricuspid and mitral valve regurgitation, with no evidence of residual intracardiac mass or communication.

The peritoneum was opened, and the peritoneal fluid was drained.

The three masses were sent for histopathology and the results were of a Non-Hodgkin B cell Lymphoma consistent with Burkitt's Lymphoma.

The patient had a unremarkable postoperative recovery and on postoperative day 8 , chemotherapy was started on according to current children's cancer and leukaemia group (CCLG) guidelines for B-cell type non-Hodgkin's lymphoma with a combination of Prednisolone, Cytarabine, Cyclophosphamide, Doxorubicin, Vincristine and intrathecal hydrocortisone and Methotrexate.

\section{Discussion:}

Primary cardiac tumours are rare, and most of them are benign neoplasms. Myxomas are the most prevalent, with fibromas, lipomatous tumours, fibroelastic papillomas, and hamartomas rounding out the list (1-2). Malignant primary cardiac tumours are uncommon, with the majority of them being sarcomas. Primary cardiac lymphomas are exceedingly rare; however, they are fast-growing and potentially curable, unlike most other cardiac malignancies (5-8). In paediatric literature, few case reports of primary cardiac lymphoma in children were published (9-12).

Clinically, lymphoma of the heart is rarely recognised. The difficulty in diagnosing it is due to its rarity and wide range of clinical symptoms. Therefore, a high level of suspicion is required in the differential diagnosis. The clinical manifestation varies depending on the location, size, and rate of growth. Congestive heart failure, chest discomfort, pericarditis, severe dyspnoea, and arrhythmia are the most common signs and symptoms.

Chemotherapy is the mainstay treatment for PCL (13-17), and it might be paired with surgical resection that showed to improve the survival (18) or radiation therapy, albeit it doesn't seem to have any influence on survival (19).

Our patient presented with short history of abdominal symptoms with watery stool, most likely the result of increased right heart filling pressures causing visceral congestion. She also complained of shortness of breath with fatigue in addition to history of migraine and rapidly progressing visual disturbances over short period.

Transthoracic echocardiography was diagnostic for 3 intracardiac masses, 2 of them were protruding into the mitral and tricuspid valves leading to severe left and right inflow obstruction, respectively. Therefore, the patient underwent urgent surgical excision of the intracardiac masses. Chemotherapy started as soon as the histopathology results were received. 
In conclusion, primary cardiac Burkitt's lymphoma is an extremely rare and fast-growing cardiac malignancy. A high index of suspicion should be maintained taking into consideration the rarity of that heart malignancy. However, cure can be achieved with combined surgical resection and multiagent chemotherapy.

\section{Conclusion:}

This case contributes to the literature in diagnosis and surgery of a rare case of primary intracardiac Burkitt Lymphoma in a child.

IRB approval, consent statement and clinical trial registration are not applicable for this case report.

\section{Conflict of interests:}

The authors declare that there is no conflict of interests.

\section{References:}

1. J.-G. Wang, H. Liu, W.-J. Yu, Y.-J. Li, and F.-J. Xin, "Primary cardiac neoplasms: a clinicopathologic analysis of 81 cases," Chinese Journal of Pathology, vol. 41, no. 12, pp. 808-812, 2012.

2. N. Kumar, S. Agarwal, A. Ahuja, P. Das, B. Airon, and R. Ray, "Spectrum of cardiac tumors excluding myxoma: experience of a tertiary center with review of the literature," Pathology Research and Practice, vol. 207 , no. 12 , pp. $769-774,2011$.

3. J. M. Sarjeant, J. Butany, and R. J. Cusimano, "Cancer of the heart: epidemiology and management of primary tumors and metastases," American Journal of Cardiovascular Drugs, vol. 3, no. 6, pp. 407-421, 2003.

4. Petrich A, Cho SI, Billett H: Primary cardiac lymphoma: An analysis of presentation, treatment, and outcome patterns. Cancer, 2011; 117(3): 581-89

5. C. E. Miguel and R. B. Bestetti, "Primary cardiac lymphoma," International Journal of Cardiology, vol. 149, no. 3, pp. 358-363, 2011.

6. L. Antoniades, C. Eftychiou, P. M. Petrou, A. Bagatzounis, and M. Minas, "Primary cardiac lymphoma: case report and brief review of the literature," Echocardiography, vol. 26, no. 2, pp. 214-219, 2009.

7. R. M. Gowda and I. A. Khan, "Clinical perspectives of primary cardiac lymphoma," Angiology, vol. 54,no. 5, pp. 599-604, 2003.

8. J. M. Sarjeant, J. Butany, and R. J. Cusimano, "Cancer of the heart: epidemiology and management of primary tumors and metastases," American Journal of Cardiovascular Drugs , vol. 3, no. 6, pp. 407-421, 2003.

9. Bassi D, Lentzner BJ, Mosca RS, Alobeid B. Primary cardiac precursor B lymphoblastic lymphoma in a child: a case report and review of the literature. Cardiovasc Pathol. 2004 Mar-Apr;13(2):116-9. doi: 10.1016/S1054-8807(03)00131-5. PMID: 15033162.

10. Agarwala B, Rubin CM. Intracardiac lymphoma in a child: successful treatment with chemotherapy alone. Pediatr Cardiol. 2000 Jul-Aug;21(4):401-2. doi: 10.1007/s002460010093. PMID: 10865026.

11. Papadopoulou AL, Argiriou M, Bonoris M, et al. Ki-1 lymphoma with cardiac involvement at initial presentation. Pediatr Hematol Oncol 1998;15:265-269.

12. Koca Yozgat A, Ozyoruk D, Kacar D, Cetin II, Yaman Bajin I, Inan K, Oguz AS. Burkitt Lymphoma Presenting With Intracardiac Mass and Tumor Thrombosis in the Anterior Mediastinum With Literature Review. J Pediatr Hematol Oncol. 2019 Apr;41(3):e197-e200. doi: 10.1097/MPH.0000000000001327. PMID: 30299348.

13. Papadopoulou AL, Argiriou M, Bonoris M, et al. Ki-1 lymphoma with cardiac involvement at initial presentation. Pediatr Hematol Oncol 1998;15:265-269. 
14. Rolla G, Bertero MT, Pastena G, et al. Primary lymphoma of the heart. A case report and review of the literature. Leuk Res 2002;26:117-120.

15. Beckwith C, Butera J, Sadaniantz A, et al. Diagnosis in oncology. Case 1: Primary transmural cardiac lymphoma. J Clin Oncol 2000; 18:1996-1997.

16. Enomoto S, Abo T, Sugawara T, et al. Successful treatment of two patients with primary cardiac malignant lymphoma. Int J Hematol 1999;70:174-177.

17. Nagano M, Uike N, Suzumiya J, et al. Successful treatment of a patient with cardiac lymphoma who presented with a complete atrioventricular block. Am J Hematol 1998;59:171-174.

18. Davis JS, Allan BJ, Perez EA, Neville HL, Sola JE. Primary pediatric cardiac malignancies: the SEER experience. Pediatr Surg Int. 2013 May;29(5):425-9. doi: 10.1007/s00383-013-3261-4. Epub 2013 Jan 29. PMID: 23358917.

19. Liang R, Yu CM, Au WY, et al. Diagnosis in oncology. Case 2: Secondary lymphoma of the heart manifesting as intracavitary masses. J Clin Oncol 2000;18:1998-1999.

Figure 1: TTE showing 3 intracardiac masses

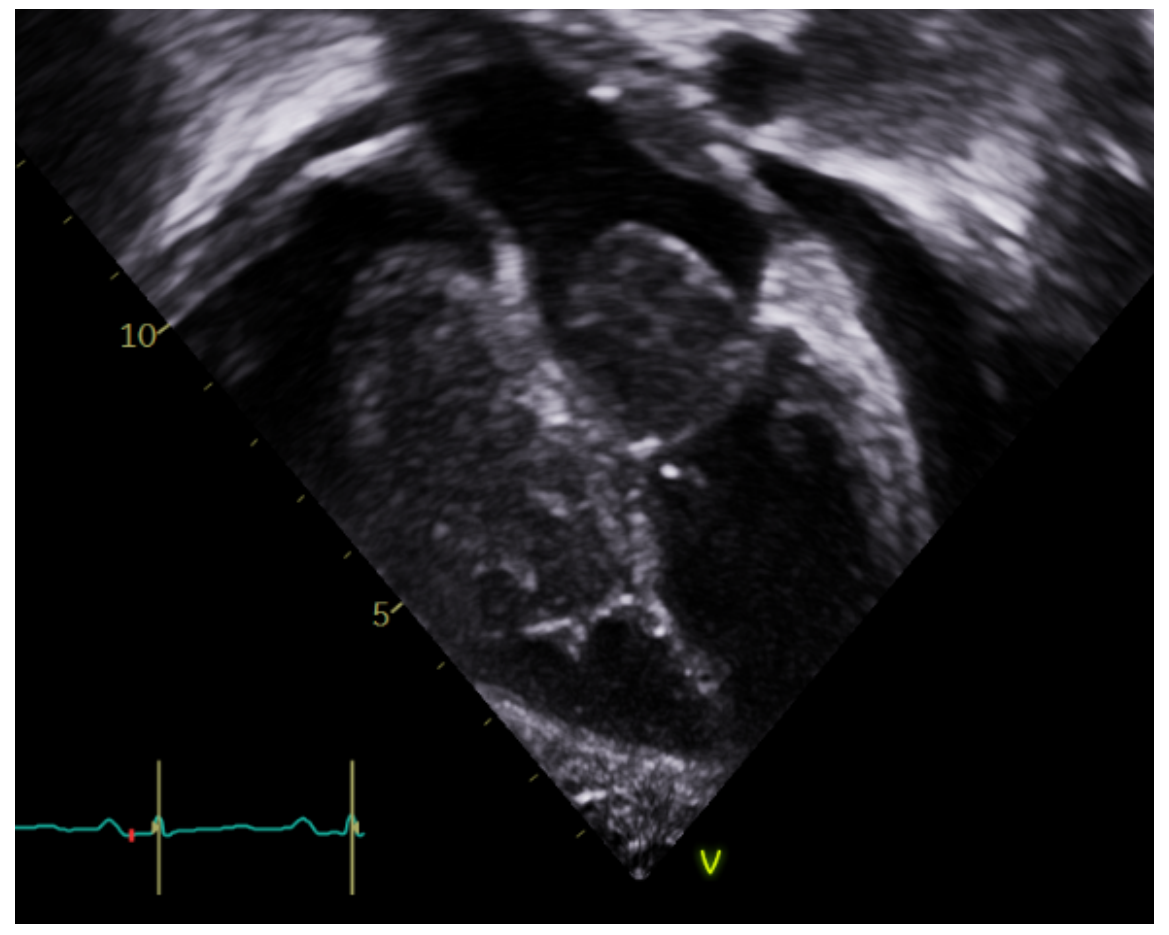

Figure 2: TTE showing 3 intracardiac masses 

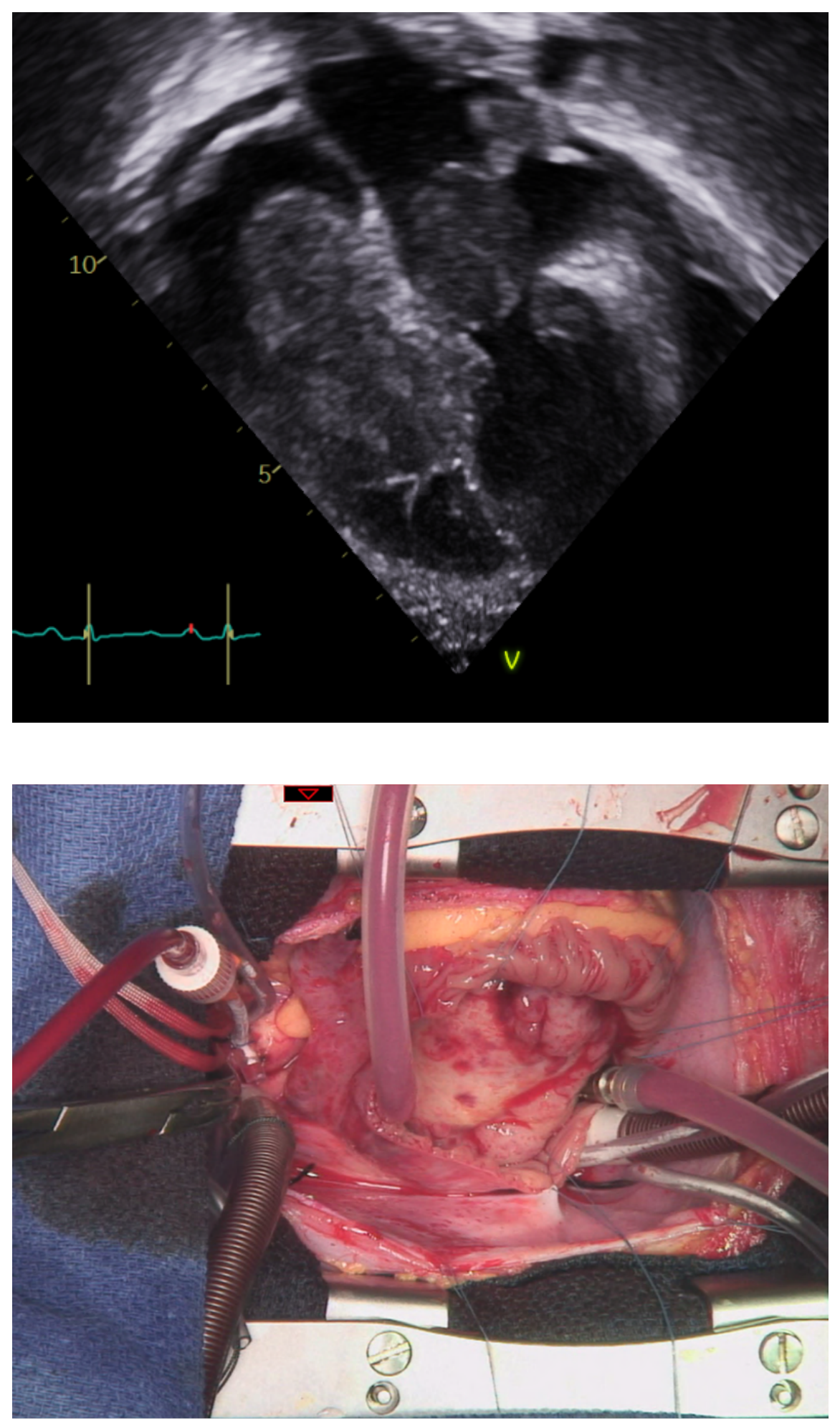

Figure 3: Intracardiac mass occluding most of the right atrium

Figure 4: Excision of the right atrial mass 

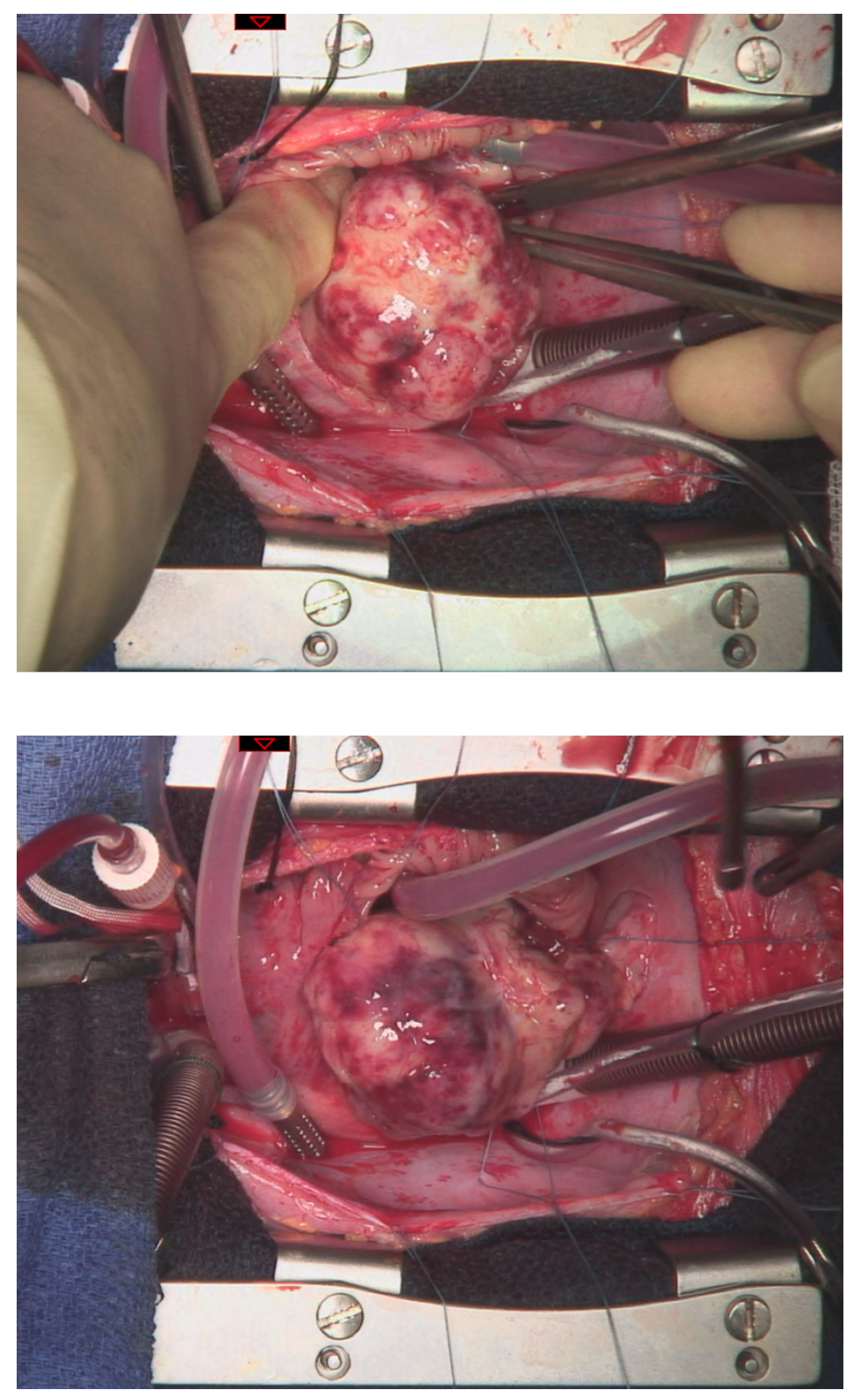


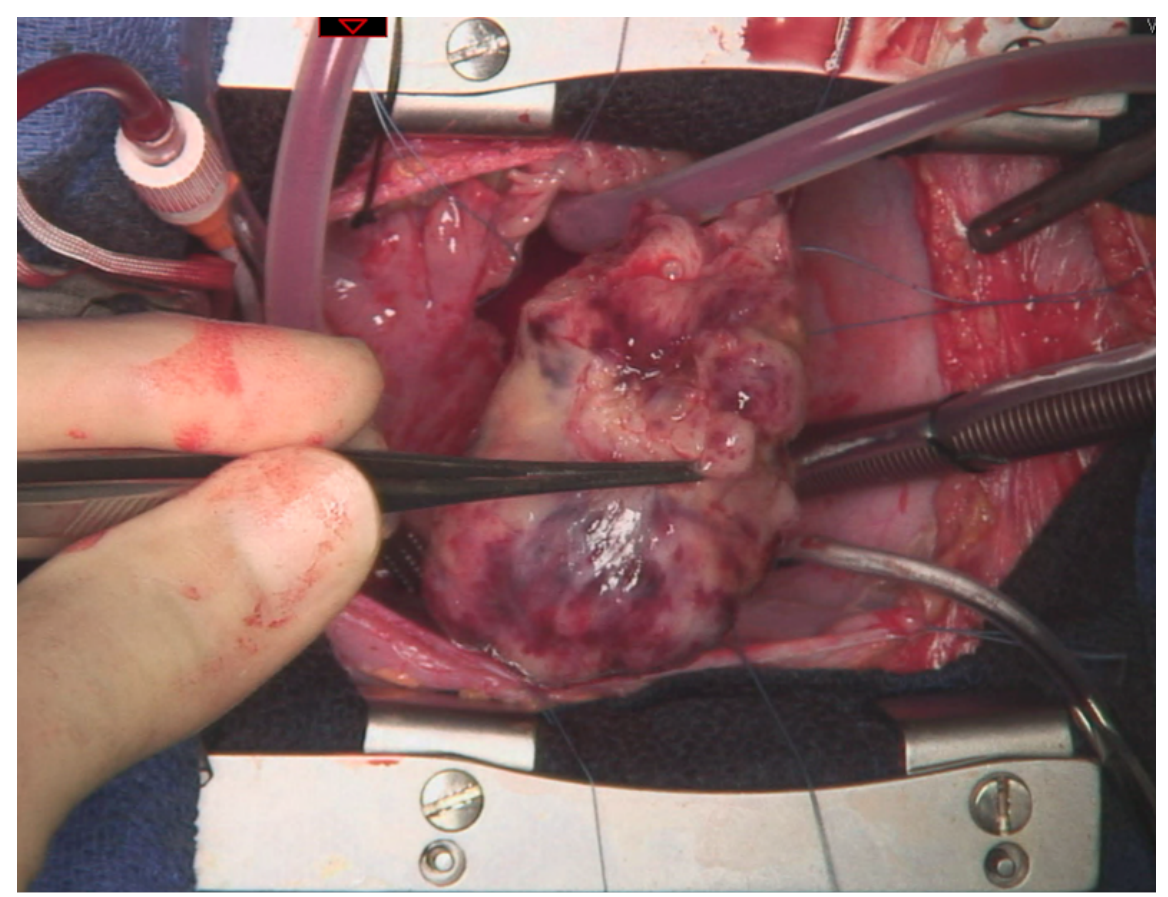

Figure 5: Left atrial mass Figure 6: Excision of the left atrial mass 